\title{
Streben nach Macht fern von Ethik: Die ,dunkle Triade“ bei Führungskräften und die Folgen für Organisationen
}

\author{
Sandra Julia Schiemann · Eva Jonas
}

Online publiziert: 21. April 2020

(C) Der/die Autor(en) 2020

Zusammenfassung Die dunkle Triade - bestehend aus Narzissmus, Machiavellismus und Psychopathie - ist mit stark unethischen Verhaltensweisen verknüpft. Personen mit hohen Ausprägungen können dementsprechend zu vielen negativen Folgen für eine Organisation führen. In diesem Artikel wird die dunkle Triade mit ihren Risiken genauer beleuchtet und die Relevanz einer ethischen Unternehmenskultur betont, die bereits bei der Stellenbesetzung beginnen sollte. Der Artikel bietet eine Übersicht über aktuelle Forschungsergebnisse zur dunklen Triade in Organisationen und ergänzt diese durch Interviews aus Wissenschaft und Wirtschaft.

Schlüsselwörter Dunkle Triade $\cdot$ Narzissmus · Machiavellismus · Psychopathie . Organisation $\cdot$ Führung $\cdot$ Ethik

\section{Striving for power far off from ethics: The "dark triad" traits among executives and the consequences for organizations}

\begin{abstract}
The dark triad-narcissism, Machiavellianism, and psychopathy-is related to unethical behavior. Therefore, people high in the dark triad traits can lead to disastrous consequences for an organization. In this article, we shed light on the dark triad and its risks as well as highlight the importance of establishing an ethical organizational culture, which should include select-out recruitment criteria. We give an overview of current research on the dark triad within organizations and complement this overview with interviews.
\end{abstract}

Dr. S. J. Schiemann $(\bowtie) \cdot$ Prof. Dr. E. Jonas

Fb. Psychologie, Universität Salzburg, Hellbrunnerstraße 34, 5020 Salzburg, Österreich

E-Mail: sandra.schiemann@sbg.ac.at

Prof. Dr. E. Jonas

E-Mail: eva.jonas@sbg.ac.at 
Keywords Dark Triad · Narcissism · Machiavellianism · Psychopathy ·

Organization $\cdot$ Leyadership $\cdot$ Etyhics

\section{Einführung}

Durch verschiedene Artikel in den Medien ist „Narzissmus“ bereits ein Begriff für viele Menschen (einige Beispiele: Faller 2019; Machac 2019; Szavarsky und Steinkogler 2019). Jedoch ist der Narzissmus - hier als subklinisch, d.h. als nicht klinisch auffällig verstanden - nur eine von drei Persönlichkeitsakzentuierungen, die zusammen in der Wissenschaft als „dunkle Triade“ bezeichnet werden. Weitere Teile der Triade sind der Machiavellismus und die subklinische Psychopathie. Diese drei sind zwar unterscheidbare, aber stark miteinander zusammenhängende Konstrukte, die mit unethischem, unmoralischem und selbstbezogenem Verhalten einhergehen (Lee und Ashton 2005; Paulhus und Williams 2002). Zudem zeigen Personen mit hohen Tendenzen der dunklen Triade allesamt geringe Ausprägungen auf dem Persönlichkeitsmerkmal „Verträglichkeit“ - also Höflichkeit, Flexibilität, Vertrauenswürdigkeit, Kooperationsbereitschaft und Toleranz (Barrick und Mount 1991; Lee und Ashton 2005). Die drei haben also einen gemeinsamen dunklen Kern, der von Moshagen et al. (2018) auch als D-Faktor bezeichnet wird: ein Streben nach dem eigenen Vorteil - auch ,über Leichen gehend“ (vgl. Bertl et al. 2017). In anderen Worten, es ist sehr wahrscheinlich, dass eine Person nicht nur hohe narzisstische Tendenzen aufweist, sondern dies auch mit Machiavellismus und Psychopathie einhergehen kann. Obwohl die drei Konstrukte einen geteilten Kern haben, unterscheiden sie sich in ihren Eigenschaften (s. Abb. 1).

Personen mit narzisstischen Tendenzen streben nach Bewunderung und zeigen sich hypersensitiv und verteidigend in Bezug auf ihr Selbstbild (Mathieu und StJean 2013; Morf und Rhodewalt 2001; Paulhus und Williams 2002; Rosenthal und

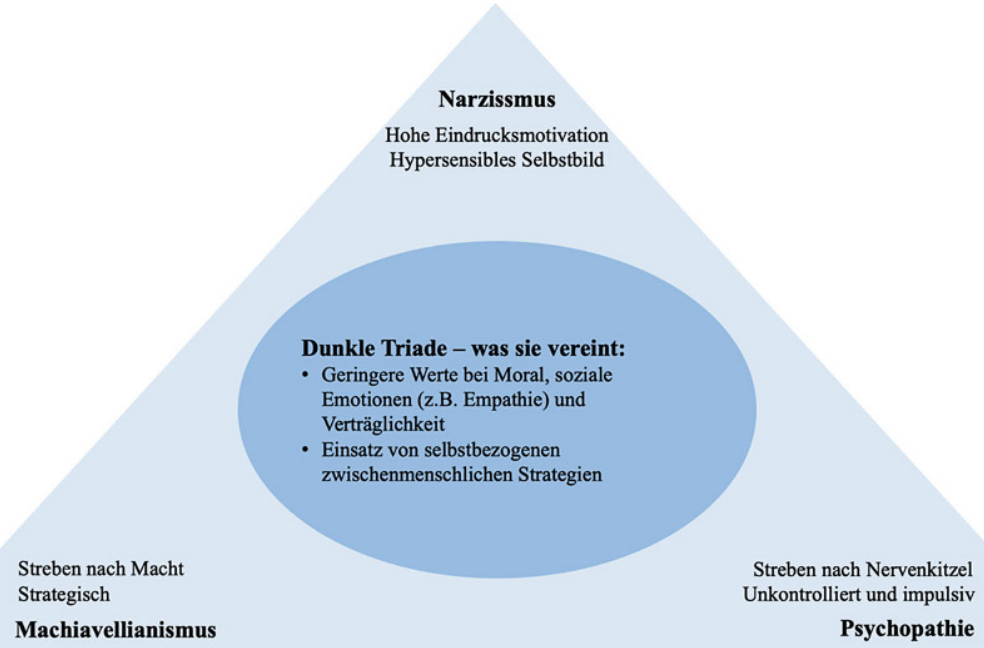

Abb. 1 Gemeinsame und distinktive Merkmale von Narzissmus, Machiavellismus und Psychopathie 
Pittinsky 2006). Diesen Personen ist es sehr wichtig, wie andere sie sehen, was den Narzissmus von den sogenannten Maliziösen Zwei - dem Machiavellismus und der subklinischen Psychopathie - unterscheidet (Kowalski et al. 2018; Rauthmann und Kolar 2012). Personen mit machiavellistischen Tendenzen streben nach Macht und nicht nach Bewunderung - um mehr Macht zu gewinnen, verwenden sie Strategien, die so wirken könnten, als wäre ihnen die Meinung anderer wichtig, jedoch ist dies nur eine Strategie (Christie und Geis 1970; Paulhus und Williams 2002). Auch Personen mit psychopathischen Tendenzen halten nicht viel von der Meinung anderer und würden für etwas Nervenkitzel jegliche Strategie ohne Schuldgefühle, Gewissensbisse oder Empathie anwenden (Babiak und Hare 2006): Während eine Person mit hohen narzisstischen Tendenzen vor anderen ihr Gesicht wahren möchte, zeigen Menschen mit hohen psychopathischen Tendenzen ihre Gleichgültigkeit gegenüber einer leidenden Person offen (Rauthmann 2011; Lee und Gibbons 2017). Aufgrund ihres Strebens nach Nervenkitzel zeigen sich Personen mit hohen psychopathischen Tendenzen impulsiv und risikofreudig (Akhtar et al. 2013; Jones und Paulhus 2011; Paulhus und Williams 2002). Zusammengefasst besteht die dunkle Triade aus Narzissmus, Machiavellismus und Psychopathie - drei Konstrukte mit einem gemeinsamen unethischen Kern. Daraus ergibt sich aus Organisationsperspektive die Frage, welche beruflichen Tätigkeiten Personen mit hohen Ausprägungen besetzen und was dies für Konsequenzen hat.

\section{Der Aufstieg der dunklen Triade in Organisationen}

Bisherige Forschung hat gezeigt, dass Personen mit hohen Tendenzen zur dunklen Triade leichter in Führungspositionen gelangen (Babiak et al. 2010; Brunell et al. 2008; Grijalva et al. 2014; Harms et al. 2011; Landay et al. 2019; Wisse und Sleebos 2017). Ihre manipulativen und oft unethischen Strategien helfen ihnen, ihr Talent positiv zu verkaufen (Back et al. 2010) und die angestrebte Stelle zu erhalten (Jonason et al. 2015; Kowalski et al. 2018; Paulhus et al. 2013). Zudem können Personen mit hohen narzisstischen Tendenzen charismatisch wirken, da sie sehr von ihrem Selbst überzeugt wirken und alles dafür tun, bewundert zu werden (Rogoza und Fatfouta 2020). Kanning sagt dazu im Interview mit Jiménez (2015): „Eine wichtige Stelle im Unternehmen besetze man im Zweifel eher mit einem Bewerber, der ein bisschen zu selbstbewusst und dominant sei, als mit einem, der zu wenig von beidem habe."

Eine weitere Fähigkeit bei Personen mit hohen Ausprägungen der dunklen Triade besteht darin, die Emotionen und Gedanken anderer gut zu verstehen, auch wenn sie selbst nicht mitfühlen und sie das kalt lässt - sie können also die Emotionen und Gedanken des Gegenübers sehr gut analysieren und somit gut manipulieren (Turner et al. 2019). Becker (2011) behauptet sogar, dass ein gewisses Maß an Ausprägung der dunklen Triade bei einer Führungskraft erwünscht ist: „Kaum jemand in einer Führungsposition kann sich von der dunklen Triade gänzlich freimachen. Ein gewisses Maß dieser Charakteristika scheint ManagerInnen überhaupt erst zu ermöglichen, ihre Rolle richtig auszufüllen." Neueste Forschung zeigt, dass Personen mit höheren Tendenzen der dunklen Triade nicht nur leichter eine Führungsposition 
erlangen, sondern auch immer weiter in der Führungsebene aufsteigen (Schiemann et al. 2020a). Schiemann et al. (2020a) erklären dies mit der starken Motivation nach Macht und Bewunderung, die diese Personen antreibt, immer höhere, mächtigere und angesehenere Positionen zu erreichen. Die Frage stellt sich nun, ob dieser Aufstieg positive oder eher negative Folgen für eine Organisation mit sich bringt.

\section{Negative Folgen für die Organisation}

Auch wenn einige Strategien dieser Personen in einem gewissen Maße einem Unternehmen nützen können - z.B. sich gut präsentieren können und charismatisch sein (Narzissmus), strategisch und macht-maximierend handeln (Machiavellismus) oder sich trauen, hohe Risiken einzugehen (Psychopathie) -, birgt die dunkle Triade auch ihre Gefahr: Bisherige Forschung hat bereits gezeigt, dass hohe Ausprägungen solcher Persönlichkeitstendenzen sehr negative Folgen für ein Unternehmen haben können. Personen mit hohen Tendenzen zur dunklen Triade führen zu vielen verschiedenen Schwierigkeiten in zwischenmenschlichen Beziehungen und schaden dem Wohlbefinden aller in einer Organisation (DeShong et al. 2015; Southard et al. 2015; Volmer et al. 2016).

So überschätzen z. B. Narzissten meistens ihre Kompetenzen (Ames und Kammrath 2004), treffen Entscheidungen auf Basis von Eindrucksmotivation (Van Dijk und De Cremer 2006) und fühlen sich schnell angegriffen (Back et al. 2013). Dieses narzisstische Verhalten hat negative Auswirkungen nicht nur auf zwischenmenschliche Beziehungen im Unternehmen (Blair et al. 2008), sondern auch auf die Performance des Unternehmens (Chatterjee und Hambrick 2007; Judge et al. 2006). Besonders die Mitarbeiter/innen der narzisstischen Führungskräfte leiden darunter: Sie fühlen sich emotional erschöpft, angespannt und depressiv, können nicht mehr so produktiv arbeiten und sehen keine Handlungsoptionen (Ellen et al. 2017). Dabei ist der Narzissmus noch das am positivsten bewertete der drei Konstrukte (,malicious two“; Kowalski et al. 2018).

Personen mit machiavellistischen Tendenzen richten durch ihr machtorientiertes, rücksichtsloses und egoistisches Verhalten ebenso Schaden in Organisationen an (Paulhus und Williams 2002). So werden unethische Strategien angewandt (Jonason et al. 2015) und ein Verhalten gezeigt, das den Interessen des Unternehmens und deren Mitarbeiter/innen entgegensteht - wie z.B. Devianz, Rache und Aggression (Machiavellismus und kontraproduktives Arbeitsverhalten; O'Boyle et al. 2012).

Ebenso abweichend verhalten sich Personen mit psychopathischen Tendenzen, deren Mitarbeiter/innen mit dem offensichtlich antisozialen Verhalten wie Lügen, Bloßstellen oder Schaden zurechtkommen müssen (Babiak et al. 2010). So kommt es zu Mobbing, unfairem Führungsverhalten, schwierigen persönlichen Beziehungen und verschiedensten Konfliktsituationen (Boddy 2011; Scherer et al. 2013). Auch sexuelle Belästigung ist mit allen drei Faktoren der dunklen Triade verknüpft und hat bei Personen mit psychopathischen Tendenzen zusätzlich die Dimension, dem Opfer die Schuld dafür zu geben (Brewer et al. 2019).

Sitzt jedoch eine Person mit hohen Ausprägungen der dunklen Triade am Kopf des Unternehmens, kann dies zu noch schwereren Folgen als nur zu einer schlech- 
teren Unternehmensperformance führen (z.B. Patel und Cooper 2014; Wales et al. 2013). So haben Unternehmen mit narzisstischeren CEOs ein extremeres Leistungsstreben als Kultur und eine höhere Fluktuationsrate - zwei Faktoren, die das gesamte Organisationsklima extrem verschlechtern (Chatterjee und Hambrick 2007). Auch wurde herausgefunden, dass Unternehmen mit narzisstischeren CEOs öfter verklagt werden, was den Ruf des Unternehmens nach außen verschlechtert und ebenso der Arbeitsatmosphäre schaden kann (O'Reilly et al. 2018). Wisse und Sleebos (2016) haben darüber hinaus herausgefunden, dass mehr Macht zu mehr feindseligem Führungsverhalten führt und somit die negativen Konsequenzen für die Mitarbeiter verstärkt (,,abusive supervision“; Wisse und Sleebos 2016). Das bedeutet, dass Personen in höheren Führungsebenen noch stärker ihre negative Seite ausleben und somit die Mitarbeiter/innen noch stärker leiden.

\section{Schwierigkeiten in der Personalentwicklung von Personen mit hohen Tendenzen der dunklen Triade}

Eine mögliche Intervention wären Personalentwicklungsmaßnahmen, die an den Einstellungen und Werten von Personen mit hohen Tendenzen der Persönlichkeitsakzentuierung in Hinblick auf die dunkle Triade arbeiten. Personalentwicklungsmaßnahmen dienen im Unternehmen dazu, die Mitarbeiter/innen als wichtiges Kapitel weiterzuentwickeln, um so ihre Kompetenzen zu verbessern und/oder zu fördern (Neuberger 2016). Dies betrifft Maßnahmen wie Training oder Coaching, die dafür verantwortlich sind, neben fachlichen auch soziale und ethische Kompetenzen zu fördern (Garavan und McGuire 2010).

Da diese Aufgabe der Personalentwicklung wenig erforscht ist, lohnt sich vorab ein Blick in die Psychotherapieforschung, bei der es ähnlich wie im Coaching um die individuelle Förderung, jedoch noch stärker um die Heilung geht (Crowe 2017). Patient/innen mit narzisstischen und psychopathischen Persönlichkeitseigenschaften gelten hier als besonders herausfordernd, nicht nur wegen ihrer fehlenden Veränderungsbereitschaft und Werte, sondern auch aufgrund ihres zwischenmenschlichen Umgangs, der ein tragfähiges psychotherapeutisches Arbeitsbündnis erschwert (Bender 2005; Caligor et al. 2015; Olver und Wong 2015; Salekin 2002). Machiavellismus ist im klinischen Bereich nicht als eigenes Konstrukt vertreten. Wie sieht es jedoch im subklinischen Bereich aus? Viel Forschung gibt es dazu nicht, aber es scheint, dass Führungskräfte mit narzisstischen Tendenzen sich nicht so einfach in die Richtung ändern können, dass sie weniger auf sich selbst und mehr auf die anderen achten (Peterson et al. 2012).

Veränderung scheint schwierig - vielleicht können Personalentwicklungsmaßnahmen dabei helfen? Harms et al. (2011) haben sich genau diesen Möglichkeitsraum angesehen und stellen fest, dass Personen mit Anzeichen einer subklinischen dunklen Triade auch über die Zeit und über Personalentwicklungsmaßnahmen hinweg keine Veränderung in ihrer Führung oder individuellen Weiterentwicklung zeigten. Da Coaching eine sehr individuelle Personalentwicklungsmaßnahme ist (Greif 2008), könnte hier vielleicht das größte Veränderungspotential liegen. 
Erste Coaching-Studien dazu offenbaren, dass es Coaching-Klient/innen mit hohen Ausprägungen der dunklen Triade gibt und dass diese die Coaches massiv herausfordern: Coaches geraten in einen Zustand von Angst, Hemmung und Disstress, was sich auf den Coaching-Erfolg negativ auswirkt (Schiemann et al. 2020b). Coaches verhalten sich zwar im Coaching gegenüber einer Klientin mit hohen Ausprägungen der dunklen Triade verglichen mit einer neutralen Klientin gleich, berichten jedoch von Angstgefühlen und von weniger empfundener Empathie hinter der vordergründig angepassten Fassade, und sie erleben sich als weniger authentisch (Schiemann et al. 2020c).

In einem kurzen Interview mit der Beraterin, Coach und Trainerin Dr. Karin von Schumann wird klar, wie die Realität mit diesen Klient/innen aussieht. Frau Dr. von Schumann hat wie viele andere Trainer, Berater und Coaches bereits Erfahrung mit Klient/innen gesammelt, die subklinisch hohe Ausprägungen der dunklen Triade haben. Sie berichtet:

Zu Beginn ist es oft nicht möglich, die Problematik zu erkennen. Diese Klienten kommen, so jedenfalls meine Erfahrung, überwiegend nicht selbstinitiiert ins Coaching, sondern weil man es ihnen für den nächsten Karriereschritt angeraten hat, oder sie kommen aufgrund von negativem Feedback aus dem Umfeld. Eine gewisse Skepsis oder Verschlossenheit scheint daher zu Beginn durchaus nachvollziehbar. Recht schnell zeigen sich jedoch im Coaching vor allem zwei Dinge: Die Klienten können absolut kein kritisches Feedback annehmen - nicht einmal in homöopathischen Dosen. Und sie sind nicht bereit oder in der Lage zur Selbstreflexion, empfinden die Aufforderung dazu manchmal schon als Zumutung - schließlich sind die anderen ja das Problem! Meiner Erfahrung nach ist das Veränderungspotential sehr gering, und man kann als Coach kaum wirksam sein.

Prof. Dr. Dieter Frey, der als Berater, Coach und Trainer arbeitet und das LMU Center for Leadership und People Management in München leitet, hat ähnliche Erfahrungen gemacht:

Ich hatte ein Coaching mit einem Vorstandsvorsitzenden eines DAX-Unternehmens, der sowohl hohe narzisstische als auch hohe machiavellistische Tendenzen zeigte. Ich habe ihn auf Probleme seiner Organisation und anderer Vorstände angesprochen, die ihn für Misserfolge verantwortlich machten. Er war total abwehrend, sah überhaupt keine Schuld bei sich, behauptete auch, eigentlich noch nie eine Fehlentscheidung getroffen zu haben, wohl aber hätten sich im Nachhinein verschiedene Situationen anders dargestellt, als er voraussehen konnte. Dass Dinge suboptimal gelaufen sind, führt er nicht auf sein Verhalten zurück, sondern auf Fehlentscheidungen nachgeordneter Ebenen. Er hatte eine absolut rigide Grundhaltung, wie die Realität zu interpretieren ist. Es gab auch im Nachhinein nur eine Sichtweise über die Realität, nämlich die seine. Er fand keinerlei Willen, sich zu verändern.

Die Interviews unterstreichen die bereits angesprochene Veränderungsschwierigkeit von Personen mit hohen Ausprägungen der dunklen Triade, da sie die Veränderung weniger bei sich, sondern mehr bei den anderen sehen. Auch in der qualitativen 
Datenauswertung berichten Coaches von dieser Resistenz und Schuldzuschreibung; darüber hinaus werden hier noch Schwierigkeiten beschrieben, die nicht den Inhalt, sondern die Interaktion mit dem Coach betreffen: Zu diesen Schwierigkeiten gehörten manipulative Taktiken, starke Suche nach Aufmerksamkeit, fehlender Zugang zu sich selbst, hohes Misstrauen oder Machtspiele (Schiemann et al. 2020b). Zusammengefasst ist es also schwer, an der Person selbst anzusetzen. Deswegen ist eine gelebte ethisch verantwortungsvolle Unternehmenskultur wichtig.

\section{Die Wichtigkeit einer ethischen Unternehmenskultur}

Wenn es um die dunkle Triade geht, so Wisse und Sleebos (2017), dürfen die Führungskraft als Akteur und die Mitarbeiter/innen, welche unter ihr leiden, nicht allein betrachtet werden, vor allem da bisherige Studien zeigen, wie machtlos die „Opfer“ solcher Führungskräfte sind. Diese Opfer können die negativen Effekte zwar mit einem hohen Selbstwertgefühl abpuffern, die Effekte jedoch auf keine Weise vermeiden (Barelds et al. 2018; Ellen et al. 2017). Oft bleibt für sie nur die Lösungsmöglichkeit, das Unternehmen zu verlassen (z.B. Ellen et al. 2017). Daher stellen Wisse und Sleebos (2017) die Dunkle Triade des Unternehmens vor, in der die negativen Effekte der dunklen Triade auf drei Faktoren zurückgeführt werden:

1. Unternehmen, das eben solches unethisches Verhalten zulässt,

2. Führungskraft/Person mit hohen Tendenzen zur dunklen Triade als Akteur,

3. Opfer, die sich das Verhalten gefallen lassen.

Diese Dreiergruppe zeigt, dass auch das Unternehmen Verantwortung trägt, solches Verhalten zuzulassen oder sogar zu unterstützen (ebd.). Dabei geht es auch um das Nicht-Zulassen von unethischem Verhalten seitens des Unternehmens - also inwieweit manipulative oder andere unethische Strategien in einem Unternehmen geduldet oder sogar gewünscht werden. Hierzu meint Prof. Frey: „,Wer im Unternehmen unethisches Verhalten zulässt und dadurch kurzfristig Vorteile auf dem Markt hat, kann vielleicht gut essen, aber nicht mehr gut schlafen." Es ist also wichtig, als Unternehmen nicht nur nach Exzellenz, Leistung, Zahlen und Profit zu streben, sondern auch nach einer ethisch verantwortungsvollen Unternehmenskultur.

Eine ethische Unternehmemskultur bedeutet, sich der Gesamtverantwortung aller Interessensgruppen und ethischer Werte bewusst zu sein und moralisch - auf Basis der Bedürfnisse und Interessen aller Interessensgruppen und nicht nur der eigenen zu handeln (Frey et al. 2010). Bisherige Forschung zeigt, dass eine geteilte ethische und ethisch gerechte Wertekultur zu mehr ethischem Verhalten, weniger Fehlverhalten und einer positiveren Einstellung gegenüber dem Unternehmen führt (Demirtas 2015; Demirtas und Akdogan 2014; Lu und Lin 2014; Mayer et al. 2010; Neubert et al. 2013; Shin et al. 2014; Wu et al. 2014). Auch Personen mit hohen Ausprägungen der dunklen Triade zeigen weniger kontraproduktives Arbeitsverhalten, wenn sie sich von der Organisation wertgeschätzt fühlen und den Eindruck haben, dass das Unternehmen um ihr Wohl besorgt ist (Palmer et al. 2017).

Zudem betont Prof. Frey die ökonomischen Vorteile einer ethisch verantwortungsvollen Unternehmenskultur: „Ethisches Verhalten lohnt sich auf die Dauer auch 
kaufmännisch. Das sieht man bei der ganzen Diesel-Affäre, wo man kurzfristig ökonomische Vorteile hatte, aber es war auch ökonomisch eine Milchmädchenrechnung. Diese Erfahrungen, die letztlich auch die Reputation eines Unternehmens auf Dauer schädigen können, können auf jedes unethische Verhalten übertragen werden." Dass eine ethisch verantwortungsvolle Unternehmenskultur tatsächlich positive Folgen für die Exzellenz eines Unternehmens hat, zeigen Resick et al. (2011): Eine ethische Führung verbessert die Arbeitsmoral und Zusammenarbeit und erhöht Motivation und Optimismus bei den Mitarbeiter/innen. Auch Ko et al. (2017) stellen in einer Meta-Analyse fest: Ethisches Führungsverhalten fördert nicht nur Zufriedenheit, Wohlbefinden und Motivation der Mitarbeiter/innen, sondern beeinflusst auch die finanzielle Performance des Unternehmens positiv.

Eine ethische Unternehmenskultur zu etablieren, ist jedoch besonders für große Unternehmen nicht leicht, da es um eine Veränderung der Normen, Strukturen und Routinen geht (Wu et al. 2014). Denn es reicht nicht aus, ethische Werte zu kommunizieren - es geht darum, die Werte zu leben: Neben klaren Leitlinien ist es daher wichtig, Multiplikatoren vom Top-Management $\mathrm{zu}$ finden, die diese Leitlinien als Vorbilder vorleben (Frey et al. 2010; Mayer et al. 2009; Ruiz et al. 2011). Ebenso kann ein starkes internes Audit helfen, unethisches Verhalten transparenter zu machen (Arel et al. 2012).

Eine solche ethische Unternehmenskultur bedeutet zudem, bereits bei der Stellenbesetzung nicht nur select-in Kriterien (was muss ein/e Kandidat/in mitbringen) zu beachten, sondern auch select-out Kriterien (was darf ein/e Kandidat/in nicht haben) zu berücksichtigen (Wisse und Sleebos 2017). Der bisherige Fokus auf selectin Stellenbesetzungen wird auch von Hell und Schneider (2016) kritisiert: ,Was ist die dunkle Seite der Persönlichkeit? Gewissenhaft, kontaktfreudig, teamfähig Stellenprofile fokussieren auf die positiven Eigenschaften einer Person.“ Jiménez (2015, S. 1) deutet im Dialog mit Kanning zudem an, dass sich das Problem für Stellenbesetzungen höherer Hierarchieebenen verschlimmert:

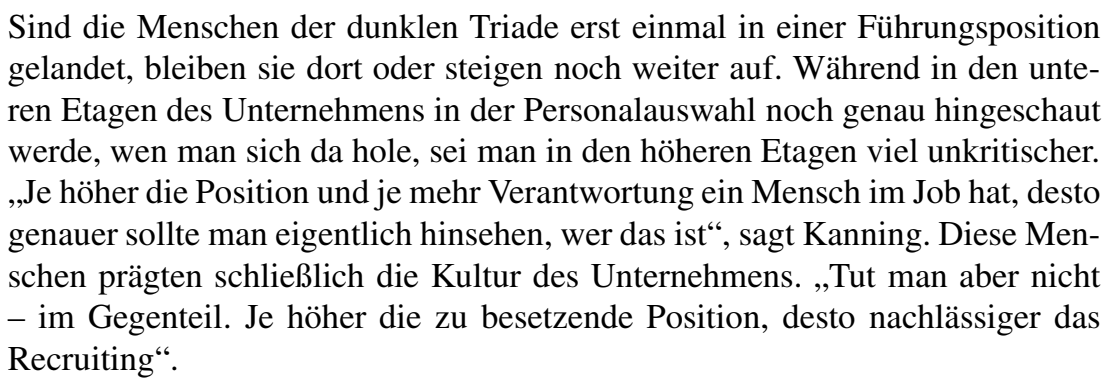

Zusammenfassend lässt sich festhalten, dass eine gelebte ethische Unternehmenskultur mit ethischen Leitlinien, Audits, Multiplikatoren aus dem Top-Management und select-out Kriterien in vor allem auch höheren internen Stellenbesetzungen die negativen Folgen durch die dunkle Triade mindern kann. So kommen wir weg vom Streben der Einzelnen fern von Ethik hin zum Streben nach Gemeinschaft mit Ethik.

Danksagung Wir danken Frau Dr. Karin von Schumann und Herrn Prof. Dr. Dieter Frey für ihre praxisnahen Einblicke im Rahmen eines kurzen Interviews zu diesem Thema. Ihre wertvollen Beiträge haben diesen Artikel wesentlich mitgeprägt. 
Funding Open access funding provided by Paris Lodron University of Salzburg.

Open Access Dieser Artikel wird unter der Creative Commons Namensnennung 4.0 International Lizenz veröffentlicht, welche die Nutzung, Vervielfältigung, Bearbeitung, Verbreitung und Wiedergabe in jeglichem Medium und Format erlaubt, sofern Sie den/die ursprünglichen Autor(en) und die Quelle ordnungsgemäß nennen, einen Link zur Creative Commons Lizenz beifügen und angeben, ob Änderungen vorgenommen wurden.

Die in diesem Artikel enthaltenen Bilder und sonstiges Drittmaterial unterliegen ebenfalls der genannten Creative Commons Lizenz, sofern sich aus der Abbildungslegende nichts anderes ergibt. Sofern das betreffende Material nicht unter der genannten Creative Commons Lizenz steht und die betreffende Handlung nicht nach gesetzlichen Vorschriften erlaubt ist, ist für die oben aufgeführten Weiterverwendungen des Materials die Einwilligung des jeweiligen Rechteinhabers einzuholen.

Weitere Details zur Lizenz entnehmen Sie bitte der Lizenzinformation auf http://creativecommons.org/ licenses/by/4.0/deed.de.

\section{Literatur}

Akhtar, R., Ahmetoglu, G., \& Chamorro-Premuzic, T. (2013). Greed is good? Assessing the relationship between entrepreneurship and subclinical psychopathy. Personality and Individual Differences, 54(3), 420-425. https://doi.org/10.1016/j.paid.2012.10.013.

Ames, D. R., \& Kammrath, L. K. (2004). Mind-reading and metacognition: Narcissism, not actual competence, predicts self-estimated ability. Journal of Nonverbal Behavior, 28(3), 187-209. https://doi.org/ 10.1023/b:jonb.0000039649.20015.0e.

Arel, B., Beaudoin, C.A., \& Cianci, A.M. (2012). The impact of ethical leadership, the internal audit function, and moral intensity on a financial reporting decision. Journal of Business Ethics, 109(3), 351-366. https://doi.org/10.1007/s10551-011-1133-1.

Babiak, P., \& Hare, R.D. (2006). Snakes in suits: when psychopaths go to work. New York: Harper Collins Publishers.

Babiak, P., Neumann, C.S., \& Hare, R.D. (2010). Corporate psychopathy: talking the walk. Behavioral Sciences \& the Law, 28, 174-193. https://doi.org/10.1002/bsl.925.

Back, M.D., Küfner, A.C.P., Dufner, M., Gerlach, T.M., Rauthmann, J.F., \& Denissen, J. J. A. (2013). Narcissistic admiration and rivalry: disentangling the bright and dark sides of narcissism. Journal of Personality and Social Psychology, 105(6), 1013-1037. https://doi.org/10.1037/a0034431.

Back, M.D., Schmukle, S. C., \& Egloff, B. (2010). What makes narcissists popular when people first meet them? PsycEXTRA Dataset. https://doi.org/10.1037/e609602010-006.

Barelds, D. P.H., Wisse, B., Sanders, S., \& Laurijssen, L. M. (2018). No regard for those who need it: the moderating role of follower self-esteem in the relationship between leader psychopathy and leader self-serving behavior. Frontiers in Psychology. https://doi.org/10.3389/fpsyg.2018.01281.

Barrick, M.R., \& Mount, M. K. (1991). The big five personality dimensions and job performance: a metaanalysis. Personnel Psychology, 44(1), 1-26. https://doi.org/10.1111/j.1744-6570.1991.tb00688.x.

Becker, L. (2011). Die dunkle Triade der Macht. Harvard Business Manager Online. https://www. harvardbusinessmanager.de/extra/artikel/a-589040.html. Zugegriffen: 14. Febr. 2020.

Bender, D. S. (2005). The therapeutic alliance in the treatment of personality disorders. Journal of Psychiatric Practice, 11(2), 73-87. https://doi.org/10.1097/00131746-200503000-00002.

Bertl, B., Pietschnig, J., Tran, U.S., Stieger, S., \& Voracek, M. (2017). More or less than the sum of its parts? Mapping the dark triad of personality onto a single dark core. Personality and Individual Differences, 114, 140-144. https://doi.org/10.1016/j.paid.2017.04.002.

Blair, C.A., Hoffman, B.J., \& Helland, K. R. (2008). Narcissism in organizations: a multisource appraisal reflects different perspectives. Human Performance, 21(3), 254-276. https://doi.org/10.1080/ 08959280802137705.

Boddy, C.R. (2011). Corporate psychopaths. London: Palgrave Macmillan. https://doi.org/10.1057/ 9780230307551.

Brewer, G., Lyons, M., Perry, A., \& O’Brien, F. (2019). Dark triad traits and perceptions of sexual harassment. Journal of Interpersonal Violence. https://doi.org/10.1177/0886260519827666. 
Brunell, A. B., Gentry, W. A., Campbell, W. K., Hoffman, B. J., Kuhnert, K. W., \& DeMarree, K. G. (2008). Leader emergence: the case of the narcissistic leader. Personality and Social Psychology Bulletin, 34(12), 1663-1676. https://doi.org/10.1177/0146167208324101.

Caligor, E., Levy, K. N., \& Yeomans, F. E. (2015). Narcissistic personality disorder: Diagnostic and clinical challenges. American Journal of Psychiatry, 172(5), 415-422. https://doi.org/10.1176/appi.ajp.2014. 14060723.

Chatterjee, A., \& Hambrick, D.C. (2007). It's all about me: narcissistic chief executive officers and their effects on company strategy and performance. Administrative Science Quarterly, 52(3), 351-386. https://doi.org/10.2189/asqu.52.3.351.

Christie, R., \& Geis, F. L. (1970). Studies in machiavellianism. New York: Academic Press. https://doi.org/ 10.1016/c2013-0-10497-7.

Crowe, T. (2017). Coaching and psychotherapy. In T. Bachkirova, G. Spence \& D. Drake (Hrsg.), The sage handbook of coaching (S. 85-101). London: SAGE.

Demirtas, O. (2015). Ethical leadership influence at organizations: evidence from the field. Journal of Business Ethics, 126(2), 273-284. https://doi.org/10.1007/s10551-013-1950-5.

Demirtas, O., \& Akdogan, A. A. (2014). The effect of ethical leadership behavior on ethical climate, turnover intention, and affective commitment. Journal of Business Ethics, 130(1), 1-9. https://doi.org/10. 1007/s10551-014-2196-6.

DeShong, H.L., Grant, D. M., \& Mullins-Sweatt, S. N. (2015). Comparing models of counterproductive workplace behaviors: the Five-Factor Model and the dark triad. Personality and Individual Differences, 74, 55-60. https://doi.org/10.1016/j.paid.2014.10.001.

Van Dijk, E., \& De Cremer, D. (2006). Self-benefiting in the allocation of scarce resources: leader-follower effects and the moderating effect of social value orientations. Personality and Social Psychology Bulletin, 32(10), 1352-1361. https://doi.org/10.1177/0146167206290338.

Ellen, B.P., Kiewitz, C., Garcia, P.R.J.M., \& Hochwarter, W. A. (2017). Dealing with the full-of-selfboss: Interactive effects of supervisor narcissism and subordinate resource management ability on work outcomes. Journal of Business Ethics, 157(3), 847-864. https://doi.org/10.1007/s10551-0173666-4.

Faller, H. (2019). Verrückt nach mir. Zeit Magazin. https://www.zeit.de/zeit-magazin/2019/33/narzissmusegoismus-persoenlichkeitsstoerung-psychologie-therapie. Zugegriffen: 14. Febr. 2020.

Frey, D., Nikitopoulos, A., Peus, C., Weisweiler, S., \& Kastenmüller, A. (2010). Unternehmenserfolg durch ethikorientierte Unternehmens- und Mitarbeiterführung. In U. Meier \& B. Sill (Hrsg.), Führung. Macht. Sinn. Ethos und Ethik für Entscheider in Wirtschaft, Gesellschaft und Kirche (S. 637-656). Regensburg: Pustet.

Garavan, T.N., \& McGuire, D. (2010). Human resource development and society: Human resource development's role in embedding corporate social responsibility, sustainability, and ethics in organizations. Advances in Developing Human Resources, 12(5), 487-507.

Greif, S. (2008). Coaching und ergebnisorientierte Selbstreflexion. Göttingen: Hogrefe.

Grijalva, E., Harms, P.D., Newman, D. A., Gaddis, B.H., \& Fraley, R. C. (2014). Narcissism and leadership: a meta-analytic review of linear and nonlinear relationships. Personnel Psychology, 68(1), 1-47. https://doi.org/10.1111/peps.12072.

Harms, P. D., Spain, S. M., \& Hannah, S. T. (2011). Leader development and the dark side of personality. The Leadership Quarterly, 22, 495-509. https://doi.org/10.1016/j.leaqua.2011.04.007.

Hell, B., \& Schneider, N. (2016). Was ist die dunkle Seite der Persönlichkeit? HR Today. https://www. hrtoday.ch/de/article/was-ist-die-dunkle-seite-der-personlichkeit. Zugegriffen: 14. Febr. 2020

Jiménez, F. (2015). Dunkle Triade: Warum radikal rücksichtslose Menschen weiter kommen. Welt. https:// www.welt.de/wissenschaft/article149692971/Warum-radikal-ruecksichtlose-Menschen-weiterkommen.html. Zugegriffen: 14. Febr. 2020.

Jonason, P. K., Wee, S., \& Li, N.P. (2015). Competition, autonomy, and prestige: mechanisms through which the dark triad predict job satisfaction. Personality and Individual Differences, 72, 112-116. https://doi.org/10.1016/j.paid.2014.08.026.

Jones, D., \& Paulhus, D. (2011). Fools rush in: emotional promiscuity predicts low IQ. PsycEXTRA Dataset.. https://doi.org/10.1037/e634112013-424.

Judge, T. A., LePine, J. A., \& Rich, B. L. (2006). Loving yourself abundantly: relationship of the narcissistic personality to self- and other perceptions of workplace deviance, leadership, and task and contextual performance. Journal of Applied Psychology, 91(4), 762-776. https://doi.org/10.1037/0021-9010.91. 4.762 . 
Ko, C., Ma, J., Bartnik, R., Haney, M.H., \& Kang, M. (2017). Ethical leadership: an integrative review and future research agenda. Ethics \& Behavior, 28(2), 104-132. https://doi.org/10.1080/10508422.2017. 1318069 .

Kowalski, C.M., Rogoza, R., Vernon, P.A., \& Schermer, J.A. (2018). The dark triad and the self-presentation variables of socially desirable responding and self-monitoring. Personality and Individual Differences, 120, 234-237. https://doi.org/10.1016/j.paid.2017.09.007.

Landay, K., Harms, P.D., \& Credé, M. (2019). Shall we serve the dark lords? A meta-analytic review of psychopathy and leadership. Journal of Applied Psychology, 104(1), 183-196. https://doi.org/10. 1037/ap10000357.

Lee, K., \& Ashton, M.C. (2005). Psychopathy, Machiavellianism, and narcissism in the five-factor model and the HEXACO model of personality structure. Personality and Individual Differences, 38(7), 1571-1582. https://doi.org/10.1016/j.paid.2004.09.016.

Lee, S. A., \& Gibbons, J. A. (2017). The dark triad and compassion: Psychopathy and narcissism's unique connections to observed suffering. Personality and Individual Differences, 116, 336-342. https://doi. org/10.1016/j.paid.2017.05.010.

Lu, C.-S., \& Lin, C.-C. (2014). The effects of ethical leadership and ethical climate on employee ethical behavior in the international port context. Journal of Business Ethics, 124(2), 209-223. https://doi. org/10.1007/s10551-013-1868-y.

Machac, L. (2019). Er dressierte mich wie einen Hund. Süddeutsche Zeitung. https://www.sueddeutsche. de/leben/narzissmus-beziehung-partner-liebe-1.4375995?reduced=true. Zugegriffen: 14. Febr. 2020.

Mathieu, C., \& St-Jean, É. (2013). Entrepreneurial personality: the role of narcissism. Personality and Individual Differences, 55(5), 527-531. https://doi.org/10.1016/j.paid.2013.04.026.

Mayer, D. M., Kuenzi, M., Greenbaum, R., Bardes, M., \& Salvador, R. B. (2009). How low does ethical leadership flow? Test of a trickle-down model. Organizational Behavior and Human Decision Processes, 108(1), 1-13. https://doi.org/10.1016/j.obhdp.2008.04.002.

Mayer, D. M., Kuenzi, M., \& Greenbaum, R. L. (2010). Examining the link between ethical leadership and employee misconduct: the mediating role of ethical climate. Journal of Business Ethics, 95, 7-16. https://doi.org/10.1007/s10551-011-0794-0.

Morf, C.C., \& Rhodewalt, F. (2001). Unraveling the paradoxes of narcissism: a dynamic self-regulatory processing model. Psychological Inquiry, 12(4), 177-196. https://doi.org/10.1207/s15327965pli1204_ 1.

Moshagen, M., Hilbig, B.E., \& Zettler, I. (2018). The dark core of personality. Psychological Review, 125(5), 656-688. https://doi.org/10.1037/rev0000111.

Neuberger, O. (2016). Personalentwicklung (2. Aufl.). Stuttgart: Enke.

Neubert, M.J., Wu, C., \& Roberts, J.A. (2013). The influence of ethical leadership and regulatory focus on employee outcomes. Business Ethics Quarterly, 23(2), 269-296. https://doi.org/10.5840/ beq201323217.

O'Boyle, E.H., Forsyth, D. R., Banks, G. C., \& McDaniel, M. A. (2012). A meta-analysis of the dark triad and work behavior: a social exchange perspective. Journal of Applied Psychology, 97(3), 557-579. https://doi.org/10.1037/a0025679.

Olver, M.E., \& Wong, S.C.P. (2015). Short- and long-term recidivism prediction of the PCL-R and the effects of age: A 24-year follow-up. Personality Disorders: Theory, Research, and Treatment, 6(1), 97-105. https://doi.org/10.1037/per0000095.

O'Reilly, C. A., Doerr, B., \& Chatman, J. A. (2018). „See you in court“: how CEO narcissism increases firms' vulnerability to lawsuits. The Leadership Quarterly, 29(3), 365-378. https://doi.org/10.1016/j. leaqua.2017.08.001.

Palmer, J.C., Komarraju, M., Carter, M.Z., \& Karau, S. J. (2017). Angel on one shoulder: Can perceived organizational support moderate the relationship between the Dark Triad traits and counterproductive work behavior? Personality and Individual Differences, 110, 31-37. https://doi.org/10.1016/j.paid. 2017.01.018.

Patel, P.C., \& Cooper, D. (2014). The harder they fall, the faster they rise: approach and avoidance focus in narcissistic CEos. Strategic Management Journal, 35(10), 1528-1540. https://doi.org/10.1002/smj. 2162.

Paulhus, D.L., \& Williams, K.M. (2002). The dark triad of personality: narcissism, machiavellianism, and psychopathy. Journal of Research in Personality, 36(6), 556-563. https://doi.org/10.1016/s00926566(02)00505-6.

Paulhus, D.L., Westlake, B.G., Calvez, S. S., \& Harms, P.D. (2013). Self-presentation style in job interviews: the role of personality and culture. Journal of Applied Social Psychology, 43(10), 2042-2059. https://doi.org/10.1111/jasp.12157. 
Peterson, S. J., Galvin, B. M., \& Lange, D. (2012). CEO servant leadership: exploring executive characteristics and firm performance. Personnel Psychology, 65(3), 565-596. https://doi.org/10.1111/j.17446570.2012.01253.x.

Rauthmann, J.F. (2011). Acquisitive or protective self-presentation of dark personalities? Associations among the dark triad and self-monitoring. Personality and Individual Differences, 51, 502-508. https://doi.org/10.1016/j.paid.2011.05.008.

Rauthmann, J.F., \& Kolar, G.P. (2012). How „,dark“ are the dark triad traits? Examining the perceived darkness of narcissism, machiavellianism, and psychopathy. Personality and Individual Differences, 53(7), 884-889. https://doi.org/10.1016/j.paid.2012.06.020.

Resick, C.J., Martin, G.S., Keating, M., Dickson, M.W., Kwan, H. K., \& Peng, C. (2011). What ethical leadership means to me: Asian, American, and European perspectives. Journal of Business Ethics, 101(3), 435-457. https://doi.org/10.1007/s10551-010-0730-8.

Rogoza, R., \& Fatfouta, R. (2020). Decoding the narcissism-charisma link: a facet approach. Personality and Individual Differences, 156, 109774. https://doi.org/10.1016/j.paid.2019.109774.

Rosenthal, S.A., \& Pittinsky, T.L. (2006). Narcissistic leadership. The Leadership Quarterly, 17(6), 617-633. https://doi.org/10.1016/j.leaqua.2006.10.005.

Ruiz, P., Ruiz, C., \& Martínez, R. (2011). Improving the „leader-follower“ relationship: Top manager or supervisor? The ethical leadership trickle-down effect on follower job response. Journal of Business Ethics, 99(4), 587-608. https://doi.org/10.1007/s10551-010-0670-3.

Salekin, R.T. (2002). Psychopathy and therapeutic pessimism: clinical lore or clinical reality? Clinical Psychology Review, 22(1), 79-112. https://doi.org/10.1016/s0272-7358(01)00083-6.

Scherer, K. T., Baysinger, M., Zolynsky, D., \& LeBreton, J. M. (2013). Predicting counterproductive work behaviors with sub-clinical psychopathy: beyond the five factor model of personality. Personality and Individual Differences, 55(3), 300-305. https://doi.org/10.1016/j.paid.2013.03.007.

Schiemann, S.J., Czibor, A., Restas, P., Szabo, J.E., \& Frey, D. (2020a). The „dark top“ and their work attitude: The magnitude of dark triad traits at various leadership levels and their influence on leaders' self- and other-rated work attitude. Unpublished manuscript under review.

Schiemann, S.J., Frey, D., \& Jonas, E. (2020b). Coach me if you can: The clients' leadership level's influence on their dark triad level and the dark triad level's negative impact on the coaches and coaching success. Unpublished manuscript under review.

Schiemann, S.J., Jutzi, C., Eberhard, I., Mühlberger, C., \& Jonas, E. (2020c). How client affiliation and interference influence the authenticity of a coach's empathy. Unpublished manuscript under review.

Shin, Y., Sung, S. Y., Choi, J. N., \& Kim, M. S. (2014). Top management ethical leadership and firm performance: mediating role of ethical and procedural justice climate. Journal of Business Ethics. https:// doi.org/10.1007/s10551-014-2144-5.

Southard, A. C., Noser, A. E., Pollock, N. C., Mercer, S. H., \& Zeigler-Hill, V. (2015). The interpersonal nature of dark personality features. Journal of Social and Clinical Psychology, 34(7), 555-586. https:// doi.org/10.1521/jscp.2015.34.7.555.

Szavarsky, C., \& Steinkogler, S. (2019). Narzissmus. https://www.krone.at/2052818. Zugegriffen: 14. Febr. 2020.

Turner, I.N., Foster, J.D., \& Webster, G.D. (2019). The dark triad's inverse relations with cognitive and emotional empathy: High-powered tests with multiple measures. Personality and Individual Differences, 139, 1-6. https://doi.org/10.1016/j.paid.2018.10.030.

Volmer, J., Koch, I.K., \& Göritz, A.S. (2016). The bright and dark sides of leaders' dark triad traits: effects on subordinates' career success and well-being. Personality and Individual Differences, 101, 413-418. https://doi.org/10.1016/j.paid.2016.06.046.

Wales, W. J., Patel, P.C., \& Lumpkin, G. T. (2013). In pursuit of greatness: CEO narcissism, entrepreneurial orientation, and firm performance variance. Journal of Management Studies, 50(6), 1041-1069. https://doi.org/10.1111/joms.12034.

Wisse, B., \& Sleebos, E. (2016). When the dark ones gain power: perceived position power strengthens the effect of supervisor machiavellianism on abusive supervision in work teams. Personality and Individual Differences, 99, 122-126. https://doi.org/10.1016/j.paid.2016.05.019.

Wisse, B., \& Sleebos, E. (2017). When the dark ones gain power: Perceived position power strengthens the effect of supervisor Machiavellianism on abusive supervision in work teams. Präsentation auf der EAWOP 2017 (Symposium 877-4, Dublin.

Wu, L.-Z., Kwan, H. K., Yim, F. H.-K., Chiu, R. K., \& He, X. (2014). CEO ethical leadership and corporate social responsibility: a moderated mediation model. Journal of Business Ethics, 130(4), 1-13. https:// doi.org/10.1007/s10551-014-2108-9. 


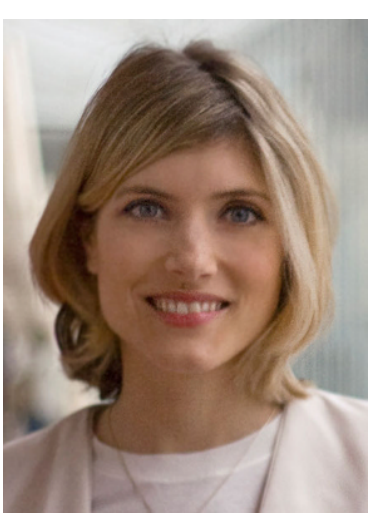

Dr. Sandra Julia Schiemann ist Senior Scientist und Senior Lecturer am Fachbereich Psychologie der Universität Salzburg, sowie Lehrende zu Organisationsentwicklung und Personalmanagement an der Privatuniversität Schloss Seeburg. Sie ist zudem zertifizierte Coach (Universität Salzburg), Mentorin (LMU Center for Leadership and People Management) und Trainerin für soziale Kompetenzen (LMU München).

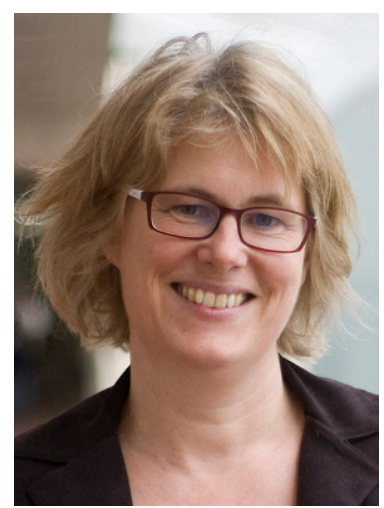

Prof. Dr. Eva Jonas leitet die Abteilung Sozialpsychologie an der Universität Salzburg. In ihrer Forschung befasst sie sich unter anderem mit motivierter sozialer Kognition im Umgang mit Bedrohungen. Darüber leitet sie sowohl den Universitätslehrgang Supervision, Coaching und Mediation als auch den Executive Master in Training und Development an der SMBS Salzburg. 